\title{
MAVKA: Investigation of stellar brightness extrema approximation stability for various methods
}

\author{
K.D. Andrych ${ }^{1,2}$, D.E. Tvardovskyi ${ }^{1,2}$, L.L. Chinarova ${ }^{1,2}$ and \\ I.L. Andronov ${ }^{2}$ \\ ${ }^{1}$ Odessa I. I. Mechnikov National University, Odessa, Ukraine, (E-mail: \\ katyaandrich@gmail.com) \\ 2 Department of Mathematics, Physics and Astronomy, Odessa National \\ Maritime University
}

Received: October 25, 2019; Accepted: January 29, 2020

\begin{abstract}
We developed the software package MAVKA for the determination of characteristics of extrema (moment of extremum, magnitude) and their errors. The program realizes the application of 11 basic functions for approximation of extrema. We tested all these methods in two parts. In the first part we used generated data sets (various smooth curves with noise). We investigated deviations between generated and computed values of moments of extremum and magnitude, as well as execution time for different extrema parameters. In the second part we used real observations of different variable stars using photometric and visual observations from different databases.
\end{abstract}

Key words: Stars: variable - photometric observations - visual observations - brightness extrema

\section{Introduction}

MAVKA is a software program for approximating moments of extrema (both maxima and minima) of stellar light curves developed by Kateryna Andrych and Ivan Andronov during the period 2015-2019 (Andrych et al., 2017; Andrych \& Andronov, 2019). Preliminary investigations of different methods were done in Andrych et al. (2015). There, photometric series of 147 stars were processed and 6509 extrema were obtained. To determine extrema with best accuracy, MAVKA is pointed to the "near-extremum" parts of the light curve. Approximations of complete eclipse shapes were compared in Andronov et al. (2017). Currently there are 9 methods included in the MAVKA algorithm of extrema fitting: algebraic polynomials in general form, "symmetric" algebraic polynomials, the New Algol Variable (NAV, Andronov, 2012), the function of Prof. Z. Mikulášek (Mikulášek, 2015), the "Asymptotic Parabola" (AP, Andronov \& Marsakova, 2006), the Wall-Supported Parabola (WSP, Andrych et al., 2017), the Wall-Supported Line (WSL), the "Wall-supported Asymptotic Parabola" 
(WSAP), the "Parabolic Spline of defect 1" (spline). Note, throughout this paper we mean both maxima and minima for the term "extrema" and times of maxima and minima for "moments of extrema."

\section{Generated data}

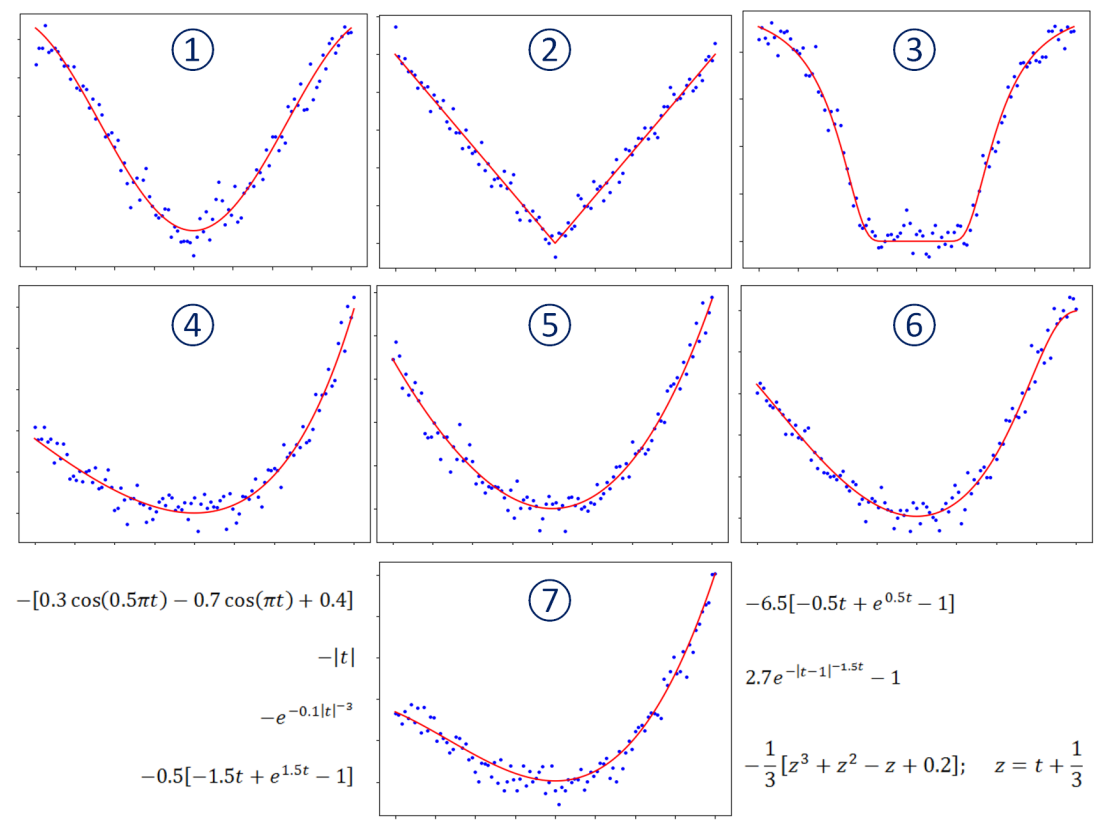

Figure 1. All minima shapes used in MAVKA. The number of the shape is the large number in the circle; for each shape, the corresponding formula is listed.

We generated 7 different shapes of minima (see Fig. 1) for testing various possible cases of the symmetric and asymmetric extrema with artificial noise. Formula (1) corresponds to a common minimum shape, formula (2) to a sharp one, formula (3) to a flat minimum. Formulas (4)-(7) are models of different shapes of asymmetric extrema.

Coefficients for all formulas correspond to the minimum position $(0,0)$ and the amplitude equals 1 . For each shape we changed the noise, number of points, and (for shapes 1-3) completeness of one or both branches. For each value of noise, number of points and degree of completeness we generated 25 extrema and processed them with the MAVKA method. Then, we obtained the average values of deviation between generated and computed moments of minima as well as magnitude. 


\section{Real observations}

MAVKA was already used in several articles: Savastru et al. (2017); Tvardovskyi et al. (2017, 2018); Andrych et al. (2020). In these papers we used the AAVSO observations of different observers. All the data were used for further O-C analyses.

Acknowledgements. We sincerely thank the organizers of the conference for the possibility to present and publish the results of this research, and the AAVSO observers for providing data.

\section{References}

Andronov, I.L.: 2012, Astrophysics, 55, 536

Andronov, I.L., Marsakova, V.I.: 2006, Astrophysics, 49, 370

Andronov, I.L., Tkachenko, M.G., Chinarova, L.L.: 2017, Astrophysics, 60, 57

Andrych, K.D., Andronov, I.L.: 2019, OEJV, 197, 65

Andrych, K.D., Andronov, I.L., Chinarova, L.L.: 2017, Odessa Astr. Pub., 30, 57

Andrych, K.D., Andronov, I.L., Chinarova, L.L., Marsakova, V.I.: 2015, Odessa Astr. Pub., 28, 158

Andrych, K.D., Andronov, I.L., Chinarova, L.L.: 2020, Journal of Physical Studies, 24, 1902, submitted (pre-print arXiv:1912.07677v2)

Chinarova, L.L., Andronov, I.L.: 2000, Odessa Astr. Pub., 13, 116

Mikulášek, Z.: 2015, Astron. Astrophys., 584, A8

Savastru, S.V., Marsakova, V.I., Andrych, K.D., Dubovsky, P.: 2017, Odessa Astr. Pub., 30, 126

Tkachenko, M.G., Andronov, I.L., Chinarova, L.L.: 2016, Journal of Physical Studies, 20, 4902

Tvardovskyi, D., Marsakova, V.I., Andronov, I.L.: 2017, Odessa Astr. Pub., 30, 135

Tvardovskyi, D., Marsakova, V.I., Andronov, I.L., Shakun, L.S.: 2018, Odessa Astr. Pub., 31, 103 